\title{
Access and benefit-sharing following the synthesis of horsepox virus
}

To the editor - In January 2018, PLOS ONE published the chemical synthesis of horsepox virus (HPXV) by a group of privately funded Canadian researchers. The authors state that "[s]ince HPXV may be extinct and the only specimen of HPXV that has been identified is unavailable for investigation, we explored whether HPXV could be obtained by large-scale gene synthesis." The DNA sequence of the HPXV genome was published ${ }^{2}$ in 2006 and is publicly available in the US National Institutes of Health GenBank database. The Canadian researchers used the HPXV genome sequence as the digital template to 'rematerialize' the virus using DNA fragments ordered from a commercial provider in Germany. Viruses from the genus Orthopox have particular genetic structures (like terminal hairpin loops) that make them difficult to both sequence and reverse engineer, but the Canadian group overcame such technical difficulties using a helper virus, recreating a "faithful copy" of the HPXV template ${ }^{1}$.

The de novo synthesis of a virus so closely related to the now eradicated smallpox virus was said to have crossed "an important Rubicon in the field of biosecurity," bringing "the world one step closer to the re-emergence of smallpox as a threat to global health security."3 In a purely technical sense, the research was simply an extension of previous synthetic biology projects that have seen the synthesis of other microorganisms like poliovirus ${ }^{4}$, the 1918 pandemic strain of H1N1 influenza virus ${ }^{5}$ and the bacterium Mycoplasma genitalium ${ }^{6}$. Scientists are starting to overcome the technical barriers associated with the de novo synthesis of simple lifeforms. The synthesis of HPXV appears to be the first time that a whole microorganism has been engineered specifically because the physical sample was not readily available to researchers as a result of regulatory constraints.

The strain of HPXV that was synthesized was based on a genetic sequence originally isolated from horses in Mongolia in 1976. This strain is now stored at the US Centers for Disease Control and Prevention (CDC) in Atlanta, USA. The CDC isolate is likely the last remaining HPXV sample as the disease it causes is thought to have been driven to extinction through modern animal husbandry practices. While it was specifically the synthesis of the HPXV that caused the controversy, this was not the ultimate intent of the Canadian research group. Their ostensible long-term aim is to create a novel alternative to the vaccinia-virus-based vaccines against smallpox.

Conflicting narratives have emerged surrounding the reasons the research group did not obtain the physical virus sample on which to conduct their vaccine research. The first, contained in the PLOS ONE article, is that the HPXV specimen was "unavailable for investigation," implying that the CDC was either unable or unwilling to provide their sample to the researchers. The second explanation is that the specimen was in fact available from the CDC subject to the terms of a material transfer agreement, but the researchers were not prepared to enter into such an agreement as they felt it might hamper their ability to commercialize their research at a later date ${ }^{7}$. In an interview with US National Public Radio the corresponding author of the study, David Evans, expressed concern that there would be future restrictions on the research they were permitted to conduct using the CDC's sample, stating that such restrictions presented "a major problem if one hopes to have any future commercial 'freedom to operate"'? It is not clear what restrictions were envisaged in this instance; however, the response to this or other concerns by the research group realizes an emerging issue: where technically possible, scientists may synthesize genetic resources to avoid real or perceived regulation on the access to physical genetic resources. As this example demonstrates, for all research leading to commercialization, the legal provenance of the materials is a key consideration. With the increasing regulation of biological materials under the evolving international regimes on access and benefit-sharing (ABS), this has become even more complicated.

\section{Virus access and benefit-sharing} In 2007 the Indonesian government cited the United Nations' 1992 Convention on Biological Diversity (CBD) when it claimed sovereignty over the H5N1 influenza virus samples that were isolated within its national borders ${ }^{8}$. This action precipitated the adoption of the World Health Organization's (WHO's) Pandemic Influenza Preparedness (PIP) Framework, which "recognize[d] the sovereign right of States over their biological resources." The recognition of viruses as "genetic resources" subject to $\mathrm{ABS}$ provisions under the $\mathrm{CBD}$ and its 2010 Nagoya Protocol on Access to Genetic Resources and the Fair and Equitable Sharing of Benefits Arising from their Utilization (Nagoya Protocol) was delayed in comparison to other genetic resources that have a clearer role in environmental conservation. Viruses are now regulated as the sovereign genetic resources of the nation state from which they were isolated, despite the viruses themselves not respecting sovereign borders, as the cliché goes. At the international level, influenza viruses with human pandemic potential are regulated as sovereign resources under the PIP Framework and all other viruses are considered sovereign genetic resources under the CBD. This notion of "viral sovereignty" may even extend to viruses that were isolated before the entry into force of the CBD'.

The CBD affirms that "the authority to determine access to genetic resources rests with national governments and is subject to national legislation." To regulate access to their sovereign genetic resources, a number of countries have implemented domestic legislative, administrative and policy measures that require potential users to obtain the prior informed consent of the providers and establish mutually agreed terms for the use of their resources. Those terms may include sharing some of the benefits of research and development with the provider of the genetic resources. The Nagoya Protocol further expanded the definition of genetic resources to include "derivatives," and while it encourages countries to implement "simplified measures on access for non-commercial research purposes," research scientists usually have to obtain consent before using genetic resources from other nations. For scientists in the biological fields, the terms "genetic resources" and "derivatives" are likely to cover just about every naturally occurring organism or part thereof, including plants, animals, fungi, spores, parasitic worms, yeasts, DNA, RNA, enzymes, biochemicals and so on.

Evans's comments to National Public Radio indicate that the HPXV researchers may have been aware of the sorts of 
limitations that might be imposed when accessing the physical sample of HPXV, including the potential requirement to engage with Mongolian authorities and share some of the eventual benefits associated with their R\&D activities. In this instance, the researchers had a clear view to commercializing a new vaccine against smallpox. Rather than negotiating access terms with the providers of the physical sample, they seemingly made the determination that it would be easier and more cost effective to recreate that sample from scratch. The chemical synthesis of HPXV was reported ${ }^{10}$ to have cost around $\$ 100,000$; that this might have been deemed more cost effective than agreeing to the terms of a material transfer agreement is a particularly concerning development for ABS. When presented with a regulatory hurdle, it appears that scientists may preferentially opt for a scientific workaround. Genetic technologies are likely to become more user-friendly and cheaper over time, so it is likely that this sort of calculus will be factored into the decisions of researchers more often.

\section{Accessing genetic sequence data} The CBD and Nagoya Protocol define genetic resources as "material," meaning that the very important intangible aspects of those resources, including genetic sequence data (GSD), are not presently within their regulatory ambit. Similarly, many of the core elements of the PIP Framework's current ABS provisions (for example, the Standard Material Transfer Agreements) apply only to "PIP biological materials" and not to the associated genetic sequence information. This means that pharmaceutical companies and other manufacturers must enter into an agreement with the WHO to access the physical pandemic influenza samples. Meanwhile, the production of influenza vaccines is no longer solely dependent on physical virus samples; they can be produced directly from viral GSD ${ }^{11}$ that is freely available online from databases like GenBank. Thus, the incentives for entering into an agreement that was designed to help the world prepare for an influenza pandemic are dramatically diminished. The same general proposition applies to other genetic resources where the use of the information has been separated from the need to possess the physical, tangible materials; this process can be described as the dematerialization of genetic resources.

The ability to synthetically recreate organisms to which ABS regulations might otherwise apply is something that the ABS community has been discussing for some time. This problem and how it might be addressed by the various ABS regulatory complexes is under active discussion, with no clear resolution in sight. Recent milestones have been the commissioning of independent research to scope the problem ${ }^{12-14}$ and submissions from interested governments and other parties ${ }^{15}$. These discussions began in the context of scientists being able to engineer single genes of interest, but we are now moving into an era that will see the ready synthesis of entire organisms. Despite these scientific advances, access to physical genetic resources will still be required, such as for the testing of the efficacy of medical countermeasures, including diagnostics, antivirals and vaccines, where synthesis costs are presently prohibitive or where certain synthesis methods are protected by intellectual property. However, as the HPXV example demonstrates, the costs and technical difficulty of virus synthesis are rapidly decreasing. Even where virus synthesis is not a complete replacement for physical genetic resources, it has significant potential to disrupt the underpinnings of current ABS regimes. In 2016, the PIP Advisory Group Technical Working Group on the sharing of influenza GSD noted that the handling of GSD "has gained importance given the recent development of synthetic biology technologies ... [which] raise questions about the broader implication of sharing and using [influenza viruses with human pandemic potential] GSD, notably with respect to benefit sharing under the PIP Framework." ${ }^{14}$ As the 2007 Indonesian example so starkly demonstrated, provider countries can stop the flow of genetic materials until their ABS concerns have been adequately addressed. Countries may also start to limit the publication of the GSD derived from their genetic resources on public repositories if they deem that benefit-sharing imperatives are not being met.

\section{Rethinking inefficient regulation}

Attempting to regulate access to physical genetic resources to direct some of the benefits associated with their use to the providing nation is an extremely inefficient form of regulation. Having what amounts to the instruction manual for recreating the physical resources published in open access databases makes capturing those benefits even more difficult. This is particularly problematic for relatively simple microorganisms like viruse ${ }^{16}$, and the WHO is probably the furthest along when it comes to considering how these issues will affect their regime for accessing influenza viruses with pandemic potential. But regulating GSD along with physical genetic resources at the upstream end of the
R\&D pipeline risks imposing more access barriers to the non-commercial research community, potentially leading to less use rather than regulatory compliance. It would also cut against the strong culture of open access in the scientific community that was reflected in the fight to keep genetic sequences available during the Human Genome Project ${ }^{17}$.

This may also be an opportunity to consider whether there are better ways of achieving the goals of ABS - the equitable distribution of benefits arising from the use of genetic resources - while ensuring access to genetic sequence data for the advancement of science, innovation and public health. The current ABS model harks back to the early 1990s, when contracts were considered to be the best instruments for agreement between those holding genetic resources and those wanting to access those physical resources. For high-value transactions and resources that have an imminent use, this was probably worth the high transaction costs of negotiating unique material transfer agreements. Most natural materials in research, however, have little immediate value, and imposing onerous obligations to negotiate, store, record and report on materials over decades has imposed a high cost on scientists with scarce research funding and time. Similar issues arise where it is not known whether a genetic resource will have imminent use but rapid distribution is a priority - such as with an emerging infectious disease with pandemic potential like COVID-19. In theory, this contractual ABS model captures the benefits for a narrow and defined public by enclosing the materials in legalities. An alternative is open access, which allows the benefits to dissipate to a broader public. Open access does away with contractual arrangements and is the current favored model for sharing and distributing GSD.

To be an equitable and acceptable alternative, open access to the physical genetic resources and continued open access to the associated GSD must also capture and share some of the benefits of their use with provider nations. One option might be taxes or levies paid not by specific user parties, but by broader publics (often governments). This would conceptualize genetic resources and associated GSD as community goods, the use of which could be funded in a similar vein to other public expenses like defense, social welfare, research and education. The CBD and Nagoya Protocol ABS regime already has a "financial mechanism" to facilitate this type of funding approach whereby developed countries could contribute funds to developing countries, although greater detail 
on how these funds would be distributed and expended will need to be considered. Similarly, an often overlooked advantage of the PIP Framework is its Partnership Contribution, which assesses annual dues for companies accessing genetic resources from the global influenza surveillance and response system and allocates those funds to pandemic preparedness and response capacities in countries that require support. These options may provide a more efficient means to capture benefits for all parties while easing the barriers to accessing physical samples and genetic sequence information. The de novo synthesis of HPXV demonstrates that scientists can and will work outside the reach of current ABS regulations because of actual or perceived costs and consequences. This serves as forewarning that, while the goals of the current ABS regimes are important, their obligations risk being perceived as overly onerous and may need to be recast in a way that does not hinder the progress of scientific research.

Editor's Note: This article has been peer-reviewed.
Michelle F. Rourke $\mathbb{D}^{1,2 凶}$, Alexandra Phelan ${ }^{3,4}$ and Charles Lawson ${ }^{5}$

${ }^{1}$ Law Futures Centre, Griffith Law School, Griffith University, Nathan, Queensland, Australia. ${ }^{2}$ CSIRO, Synthetic Biology Future Science Platform, Brisbane, Queensland, Australia. ${ }^{3}$ Center for Global Health Science and Security, Georgetown University Medical Center, Washington, USA. ${ }^{4} O$ 'Neill Institute for National and Global Health Law, Georgetown University, Washington, DC, USA. ${ }^{5}$ Environmental Futures Research Institute, Griffith Law School, Griffith University, Gold Coast, Queensland, Australia. 凶e-mail:m.rourke@griffith.edu.au

Published online: 22 April 2020 https://doi.org/10.1038/s41587-020-0518-z

References

1. Noyce, R. S., Lederman, S. \& Evans, D. H. PLoS ONE https://doi. org/10.1371/journal.pone.0188453 (2018).

2. Tulman, E.R. et al. J. Virol. https://doi.org/10.1128/JVI.00945-06 (2006).

3. Koblentz, G. D. Health Secur. 15, 620-628 (2017)

4. Cello, J., Paul, A. V. \& Wimmer, E. Science 297, 1016-1018 (2002).

5. Tumpey, T. M. et al. Science 310, 77-80 (2005).

6. Gibson, D. G. et al. Science 319, 1215-1220 (2008).

7. Greenfieldboyce, N. Did pox virus research put potential profits ahead of public safety? https://www.npr.org/sections/ health-shots/2018/02/17/585385308/did-pox-virusresearch-put-potential-profits-ahead-of-public-safety (2018).
8. Sedyaningsih, E. R., Isfandari, S., Soendoro, T. \& Supari, S. F. Ann. Acad. Med. Singapore 37, 482-488 (2008).

9. Rourke, M. F. J. Law Med. 25, 429-447 (2018).

10. Kupferschmidt, K. Science https://doi.org/10.1126/science. aan7069 (2017).

11. Dormitzer, P. R. et al. Sci. Transl. Med. 5, 185 ra68 (2013).

12. Ad Hoc Technical Expert Group on Digital Sequence Information on Genetic Resources. Fact-Finding and Scoping Study on Digital Sequence Information on Genetic Resources in the Context of the Convention on Biological Diversity and the Nagoya Protocol CBD/ DSI/AHTEG/2018/1/3. https://www.cbd.int/doc/c/b39f/4faf/7668 900e8539215e7c7710fe/dsi-ahteg-2018-01-03-en.pdf (2018).

13. Intergovernmental Technical Working Group on Forest Genetic Resources. Draft Exploratory Fact-Finding Scoping Study on 'Digital Sequence Information' on Genetic Resources for Food and Agriculture CGRFA/WG-PGR-9/18/Inf.14. http://www.fao.org/3/ CA2359EN/ca2359en.pdf (2018).

14. Pandemic Influenza Preparedness (PIP) Framework Advisory Group Technical Working Group (TWG) on the Sharing of Influenza Genetic Sequence Data. Optimal Characteristics of an Influenza Genetic Sequence Data Sharing System under the PIP Framework. http://www.who.int/influenza/pip/advisory_group/ twg_doc.pdf?ua=1 (2016)

15. Ad Hoc Technical Expert Group on Digital Sequence Information on Genetic Resources. Synthesis of Views and Information on the Potential Implications of the Use of Digital Sequence Information on Genetic Resources for the Three Objectives of the Convention and the Objective of the Nagoya Protocol CBD/ DSI/AHTEG/2018/1/2. https://www.cbd.int/doc/c/06dc/df41/ cbbe0ff3d861dc4e45953973/dsi-ahteg-2018-01-02-en.pdf (2018).

16. Gostin, L. O., Phelan, A., Stoto, M. A., Kraemer, J. D. \& Reddy, K. S. Science 345, 1295-1296 (2014).

17. Lawson, C. \& Rourke, M. J. Law Med. 24, 96-118 (2016).

Competing interests

The authors declare no competing interests. 